\title{
THE RADIATIVE IMPROVED MODE IN TEXTOR: POWER EXHAUST AND IMPROVED CONFINEMENT AT HIGH DENSITY
}

\author{
B. UNTERBERG,* U. SAMM, and M. Z. TOKAR' Institut für Plasmaphysik, Forschungszentrum Jülich GmbH \\ EURATOM Association, Trilateral Euregio Cluster, D-52425 Jülich, Germany
}

\begin{abstract}
A. M. MESSIAEN and J. ONGENA Laboratory for Plasma Physics, Ecole Royale Militaire Koninklijke Militaire School, Association EURATOM-Belgian State, Trilateral Euregio Cluster B-1000 Brussels, Belgium
\end{abstract}

R. JASPERS FOM - Institute for Plasma Physics Rijnhuizen, Association EURATOM-FOM Trilateral Euregio Cluster, P.O. Box 1207, NL-3430 BE Nieuwegein, The Netherlands

Received June 4, 2004

Accepted for Publication October 5, 2004

The concept of a cold radiating plasma boundary has been proposed as a solution to the problem of power exhaust in magnetically confined fusion plasmas. We describe experiments to study the impact of the radiating impurities on transport processes in the plasma boundary and the plasma core in the tokamak TEXTOR.

The injection of impurities (neon, silicon, or argon) leads to the formation of a radiating plasma boundary where up to $90 \%$ of the input power can be distributed to large wall areas, thereby strongly reducing the convective heat flux density onto the plasma-facing components. At high plasma densities the impurity seeding leads to a transition to an improved confinement state termed the radiative improved mode. This operational scenario combines high density and high confinement with power exhaust by radiation under quasi-stationary discharge conditions.

The confinement improvement can be explained by a reduction of transport caused by the ion temperature gradient mode. This reduction is initiated by the impurity content and amplified by a characteristic steepening of the density profiles of the background plasma. The extrapolation of the results obtained in TEXTOR, based on experiments in larger devices, is discussed.

KEYWORDS: radiative improved mode, improved confinement, impurity radiation

\section{INTRODUCTION}

With the successful demonstration of a D-T fusion plasma close to breakeven producing a fusion power of 16.1 MW in JET (Ref. 1), magnetic fusion research has resulted in the design of the next step device ITER to achieve a fusion gain $Q=P_{\text {fus }} / P_{\text {aux }}=10$ (Ref. 2). Now the issue of heat and particle (especially helium) exhaust from the fusion plasma and the problem of erosion at the plasma-facing components of the first wall in a fusion reactor come into the focus of fusion research. In this respect only an integral and coherent approach to investigate fusion plasmas will finally lead to an attractive

*B.Unterberg@fz-juelich.de scenario, namely, both the achievement of a suited fusion triple product and the accomplishment of proper solutions for particle and energy exhaust.

On one hand the helium ash generated in the fusion process has to be exhausted to prevent a quench of the process because of fuel dilution, and on the other hand the power flow out of the plasma must be moderated, which is - together with the flow of the charged plasma constituents along the magnetic field-concentrated on relatively small areas by the magnetic field. While such a concentration of the heat flux gives rise to problems with the first wall materials, a concentration of the particle flux, especially of the helium flux, is needed to allow efficient extraction of the helium ash from the system. Thus, energy and particle exhaust can be regarded 
as key issues for a stationary fusion plasma in addition to the realization of a stationary confining magnetic field itself. The lifetime of the plasma-facing components is crucial for the availability of a fusion reactor, which, finally, determines its economics to a large extent.

To solve the problem of power exhaust from fusion plasmas the concept of a cold radiating plasma mantle has been proposed. ${ }^{3,4}$ Within this concept the power flux out of the plasma is distributed to large areas by photons, which are not affected by the magnetic field. The radiation losses originate from line radiation of impurities at the plasma periphery, which are intentionally and in a restricted manner injected into the plasma discharge.

The application of such a concept to a fusion reactor is restricted to the degree to which different requirements can be met simultaneously: good energy confinement, high-density operation, efficient helium exhaust, restriction of the line radiation to the plasma edge with sufficiently low impurity concentration in the core plasma, as well as stationary and stable plasma conditions.

The first experimental proof of this concept in a tokamak plasma with reactor-relevant heat flux densities and stationary operational conditions was demonstrated at the TEXTOR device in Jülich using feedback controlled neon injection to provide the cold radiating plasma mantle. 5,6 Later on, condensing species like silicon have been investigated. ${ }^{7}$ The compatibility with helium exhaust has been examined, ${ }^{8}$ and detailed calculations of the influence of radiating impurities on the power balance in a fusion reactor were done. ${ }^{9}$

Interestingly and contrary to the common belief in early fusion research, impurity injection and the radiating plasma boundary do not decrease the energy confinement time but surprisingly can lead to a substantial improvement of confinement. First indications of such a beneficial influence of impurities on the energy transport in the plasma bulk were reported from the Impurity Study Experiment B (ISX-B) using neon seeding in auxiliary heated plasma discharges as early as 1984 (Ref. 10). In the divertor tokamak ASDEX neon seeding has been applied to recover improved ohmic confinement at high plasma densities. ${ }^{11}$

In the TEXTOR tokamak in Jülich, first observations of improved energy confinement in discharges with impurities and a radiating boundary were made in 1992 after the first wall of the device was coated with a siliconcontaining layer (so-called siliconization) ${ }^{12,13}$ leading to plasma discharges with a radiating boundary due to silicon erosion from the plasma-facing components via sputtering (see also the description in Ref. 7). Improved energy confinement could then also have been established using noble gases like neon or argon to form the radiating boundary. The new plasma regime was then termed radiative improved mode ${ }^{14-18}$ (RI-mode). At high plasma densities around the empirical Greenwald limit ${ }^{19}$ the energy confinement time can be as high as in $\mathrm{H}$-mode discharges (discharges with improved confinement owing to a trans- port barrier at the edge) without edge-localized modes (ELMs) (periodic bursts of energy and particles out of the confinement plasma).

Once a transition to the RI-mode state is obtained, the plasma density can be further raised without loss of confinement by careful external gas fueling. However, if the external gas fueling is too strong, a back transition to L-mode confinement is observed. ${ }^{20-22}$

Subsequent to the first RI-mode results in TEXTOR, the ASDEX-Upgrade team also successfully tested impurity seeding in beam heated $\mathrm{H}$-mode discharges, leading to the so-called completely detached H-mode ${ }^{23}$ (CDHmode). Discharge conditions with a radiating plasma edge have since then been obtained in various circular and elongated limiter and divertor machines, including the largest tokamaks in the world: DIII-D (Refs. 24, 25, and 26), TFTR (Refs. 27 and 28), JT-60U (Refs. 29) and JET (Refs. 30, 31, and 32). A variety of operational procedures has been used with impurity seeding, all leading to discharges with at least the same or even enhanced confinement and density as the unseeded counterparts. Promising results have been obtained with sophisticated feedback control techniques, leading to stationary plasmas at high density and confinement.

In this contribution we review the work performed in TEXTOR starting in Sec. II with a description of the radiating plasma mantle and its beneficial impact on the plasma edge properties. In Sec. III we summarize the global confinement properties in discharges with a radiating plasma mantle and improved energy confinement at high density (RI-mode) before we present our understanding of the physical mechanisms leading to the confinement improvement during the transition to the RI-mode state in Sec. IV. Section V attempts an outlook to future devices on the basis of experiments with impurity seeding performed on the large tokamak devices DIII-D and JET, and Sec. VI ends this review with a summary and conclusion.

\section{FORMATION OF A RADIATING PLASMA MANTLE AND IMPACT ON THE PLASMA EDGE PROPERTIES}

The influence of impurities on the energy and particle fluxes to the edge has been studied extensively in TEXTOR (major radius $R=1.75 \mathrm{~m}$, minor radius $a=$ $0.46 \mathrm{~m}$ defined by the toroidal pump limiter ALT-II). Substantial changes of the plasma boundary parameters can be observed when the fraction of the radiation power $\gamma$ is increased by neon seeding at densities around the Greenwald density.

Figure 1 shows the effect on the plasma edge density and temperature when the radiated power fraction is increased from $\gamma=35 \%$ to $90 \%$. The electron temperature at the last closed flux surface (LCFS) $T_{e}$, measured in the outer midplane of the poloidal cross section (at the low 


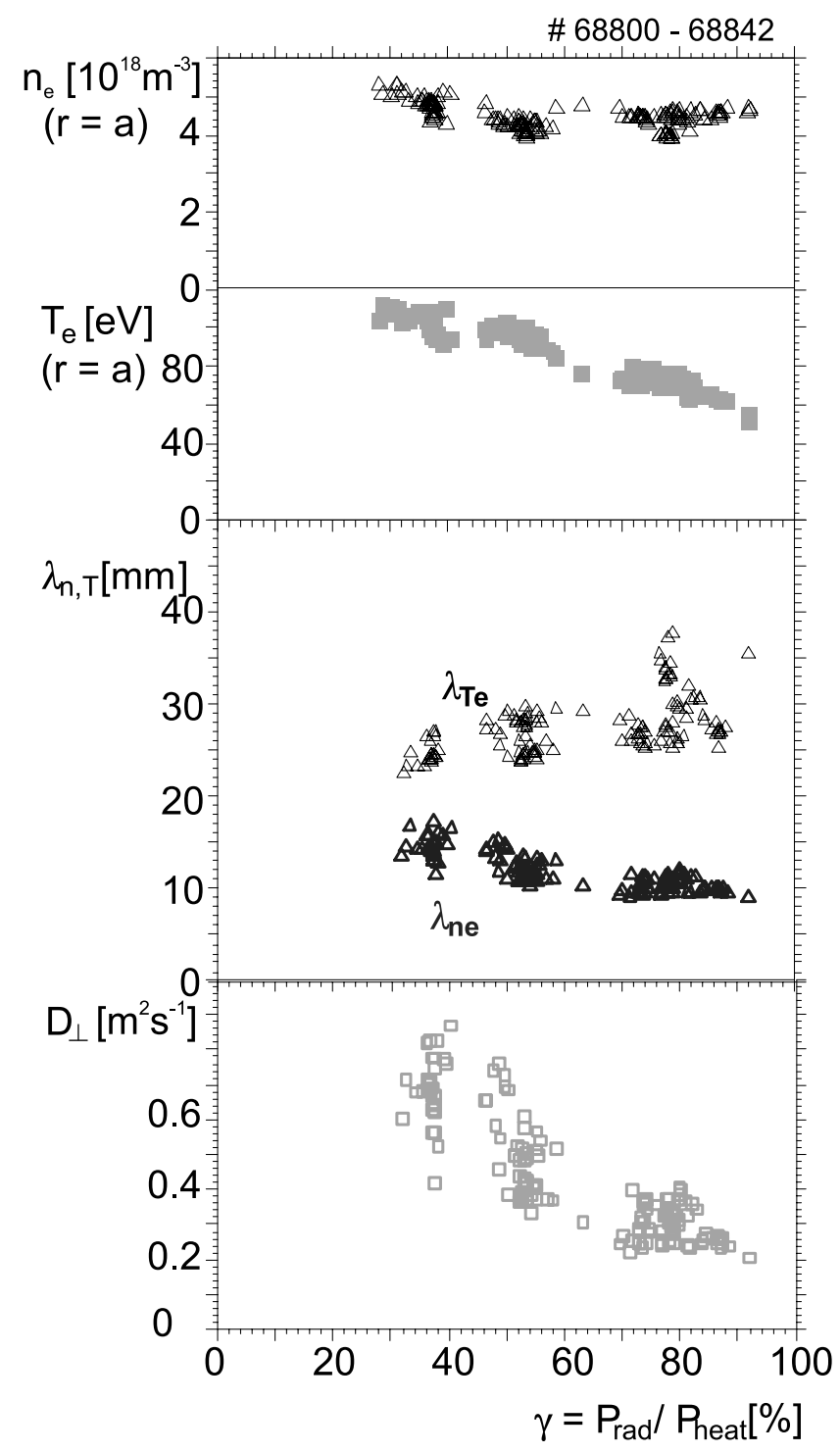

Fig. 1. Electron density $n_{e}$ and temperature $T_{e}$ at the LCFS, decay lengths of the electron density $\lambda_{n}$ and temperature $\lambda_{T}$, and diffusion coefficient $D_{\perp}$ in the SOL against radiation level $\gamma\left(\bar{n}_{e}=0.85-1.1 \cdot n_{G W}, P_{\text {heat }}=2.2-\right.$ 2.7 MW, $I_{p}=400 \mathrm{kA}$, adapted from Ref. 33).

field side), is reduced roughly by a factor of 2 from about $100 \mathrm{eV}$ in discharges without impurity injection (L-mode reference) to about $50 \mathrm{eV}$ at the highest radiation fraction. This finding documents the desired plasma boundary cooling as a consequence of the radiating plasma mantle and the reduced heat flow into the scrape-off layer (SOL). At the same time, the electron density decreases slightly by about $15 \%$. The fuel density, however, decreases much stronger owing to the substantial dilution by the seeded impurities in the edge. The decay length of the electron density $\lambda_{n}$ as determined from an exponential fit in the SOL is roughly constant and equal to $10 \mathrm{~mm}$ for this series of discharges, whereas the electron temperature profiles tend to flatten with a decay length increasing from $\lambda_{T_{e}}=25$ to about $35 \mathrm{~mm}$.

Using the electron and ion temperatures measured to calculate the ion sound velocity $c_{s}$ and the density decay length, we estimate the diffusion coefficient in the SOL according to the simple relation $D_{\perp}=\lambda_{n}^{2} c_{s} / L_{\|}$. $L_{\|}$denotes the connection length in the SOL (for TEXTOR $L_{\|}=18.6 \mathrm{~m}$ at $I_{p}=400 \mathrm{kA} / B_{T}=2.25 \mathrm{~T}$ ). This representation of the diffusion coefficient neglects the impact of ionization processes on the electron density profiles, which may cause a flattening of the density profile. The substantial decrease of $D_{\perp}$ with $\gamma$ by a factor of 3 , however, cannot solely be related to a reduction of the fraction of ionizations inside the SOL. Therefore, radiation cooling leads to a substantial decrease of particle flux out of the confined volume.

Owing to the decrease of both the electron temperature and the density at the LCFS, the penetration depth of neutrals into the confined volume increases with increasing fraction of radiated power $\gamma$. This effect has been experimentally quantified for deuterium, neon, and helium. ${ }^{17}$

These changes of the plasma boundary parameters, namely, the decrease of the diffusion coefficient and the increase of both the penetration depth and the fraction of ionizations inside the LCFS, lead to an improved particle confinement. The recycling flux $\Gamma_{r e c}$ is much larger than the external fueling rate and the rate $d N_{P} / d t$ (corresponding to a recycling coefficient $R$ close to one). Therefore, the product between particle confinement time and fueling efficiency of recycling neutrals can be given by $f_{0} \cdot \tau_{p}=$ $N_{P} / \Gamma_{\text {rec }}$, where the factor $f_{0}$ denotes the fueling efficiency of the neutrals. This quantity is shown in Fig. 2 and increases according to the reduction of $\Gamma_{r e c}$. If we allow an increase of $f_{0}$ from about 0.6 to 0.8 , as indicated by our experimental results concerning the neutral fueling efficiency, we still find a substantial increase of the particle confinement time by about a factor of 2 comparing Land RI-mode. The improved particle confinement in the RI-mode has been found to be important to maintain a high plasma density with moderate external gas input.

One drawback of the increased particle confinement is the simultaneous increase of the effective particle replacement time for helium, $\tau_{p, H e}^{*}$, which increases proportional to the helium confinement time, $\tau_{p, H e}$ (Ref. 16), in the RI-mode. This behavior is in contrast to what has been observed under L-mode conditions with radiation cooling, where the increase of $\tau_{p, H e}$ could be compensated by an increase of the exhaust efficiency in cold edge plasmas. ${ }^{8}$ Note, however, that the figure of merit for helium removal, $\rho_{H e}^{*}=\tau_{p, H e}^{*} / \tau_{E}$, does not increase because of the improvement of the energy confinement time $\tau_{E}$ when going from the unseeded L- to the impurity seeded RI-mode.

To judge the use of impurities injected to form a radiating plasma mantle, one has to address mainly two 


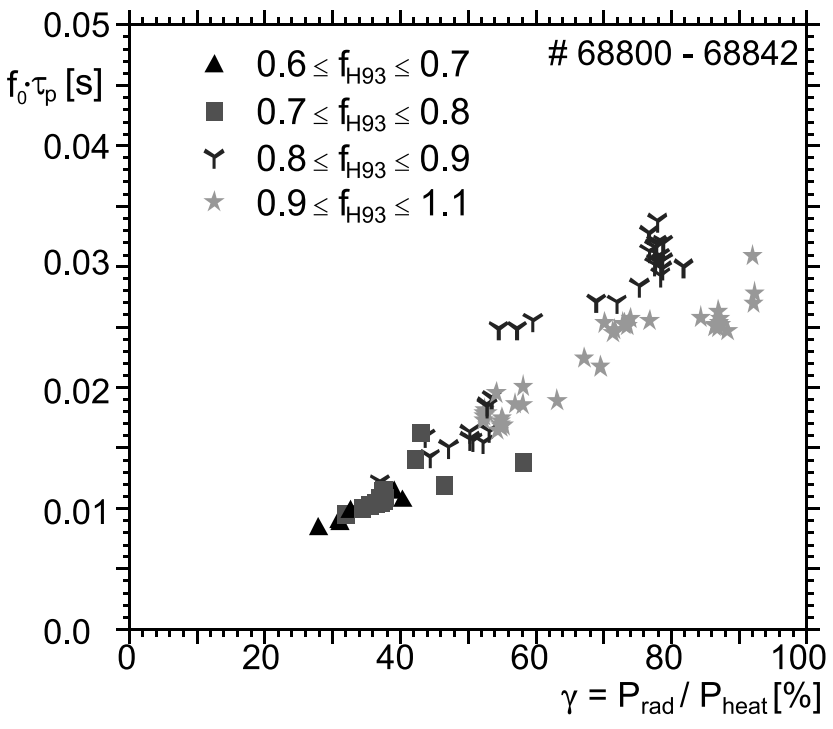

Fig. 2. Product of particle confinement time $\tau_{p}$ and the fueling efficiency of neutrals $f_{0}$ against radiation level $\gamma$. Different symbols denote different energy confinement with respect to the ELM-free H-mode $\left(\bar{n}_{e}=0.85-1.1 \cdot n_{G W}\right.$, $\left.P_{\text {heat }}=2.2-2.7 \mathrm{MW}, I_{p}=400 \mathrm{kA}\right)$.

aspects: the absolute amount of impurities in the plasma and their radial distribution and the influence of impurity seeding on intrinsic impurities (in TEXTOR mainly carbon and oxygen). The substantial reduction of deuterium flux owing to the improved particle confinement in discharges with a radiating boundary strongly affects the impurity production at the main limiter ALT-II. The absolute fluxes of carbon and oxygen are reduced with increasing $\gamma$. However, the reduction is not as high as for deuterium so that the effective yields (relation between impurity and deuterium fluxes) increase from 0.01 to 0.025 for carbon and from 0.005 to 0.02 for oxygen when $\gamma$ is raised from 35 to $85 \%$. The flux ratio between neon and deuterium needed to radiate $85 \%$ of the total input power of $2.7 \mathrm{MW}$ reaches values up to 0.05 to 0.07 . The substantial amount of neon and the increase of the effective yields of the intrinsic carbon and oxygen lead to an increased dilution at the plasma boundary. To estimate the deuterium concentration at the plasma boundary we use the measured flux ratios $\Gamma_{i} / \Gamma_{D}$ between impurity and deuterium neutrals $(i=\mathrm{Ne}, \mathrm{C}, \mathrm{O})$. The flux ratio $\Gamma_{i} / \Gamma_{D}$ is related to the ionic concentration of an impurity species $i, n_{i} / n_{D}$, by $\Gamma_{i} / \Gamma_{D}=\left(v_{i} n_{i}\right) /\left(c_{s} n_{D}\right)$, where $v_{i}$ denotes the velocity of impurity ions entering the Debye sheath in front of the limiter surface and $c_{s}$ the ion sound velocity of deuterium. Because of friction forces between impurity and deuterium ions, the ion velocity $v_{i}$ approaches $c_{s}$ at high densities and low temperatures in the SOL (Ref. 34). Therefore, we assume $\Gamma_{i} / \Gamma_{D}=n_{i} / n_{D}$ to estimate the dilution at the edge giving a lower limit for the ionic impurity concentration at the plasma edge as $v_{i}<c_{s}$. Using the condition of quasi neutrality, $n_{e}=n_{D}+$ $\sum\langle Z\rangle_{i} n_{i}$, where all impurity species $i$ have to be added up and $\langle Z\rangle_{i}$ denotes their average charge state, the deuterium concentration $n_{D} / n_{e}$ can be expressed as

$$
\frac{n_{d}}{n_{e}}=\left(1+\sum_{i}\langle Z\rangle_{i} \frac{\Gamma_{i}}{\Gamma_{d}}\right)^{-1}
$$

The deuterium concentration at the edge according to Eq. (1) is reduced from 0.9 at $\gamma=35 \%$ to $<0.7$ at $\gamma=$ $85 \%$. However, there are remarkable differences concerning the dilution in the plasma center compared to the dilution at the edge.

To compare the dilution at the plasma edge described before with the dilution of the plasma core, we calculate the central deuterium concentration $c_{D}(0)$ with the help of the carbon and neon concentration measured by charge exchange recombination spectroscopy (CXRS) on axes as shown in Fig. 3 (Ref. 35). The ratio between carbon and oxygen density in the center has been assumed to be equal to the ratio of carbon and oxygen flux at the ALTlimiter. Neon, carbon, and oxygen are assumed to be fully ionized. Using the condition for quasi neutrality we obtain a central deuterium concentration $c_{D}(0)$ only slightly decreasing from 0.86 at $\gamma=35 \%$ to 0.81 at $\gamma=$ $85 \%$. This fact can be understood by the partial replacement of carbon in the center (with central concentrations decreasing from 0.014 at $\gamma=35 \%$ to 0.01 at $\gamma=85 \%$ by neon with a concentration of 0.009 at $\gamma=85 \%$ ).

These findings are consistent with an analysis of the neutron reactivity measured in the plasma. ${ }^{36}$ The effective charge of the plasma $Z_{\text {eff }}$ in the center is calculated with the help of the impurity concentrations from the CXRS measurements and increases from 1.8 to 2.5 with

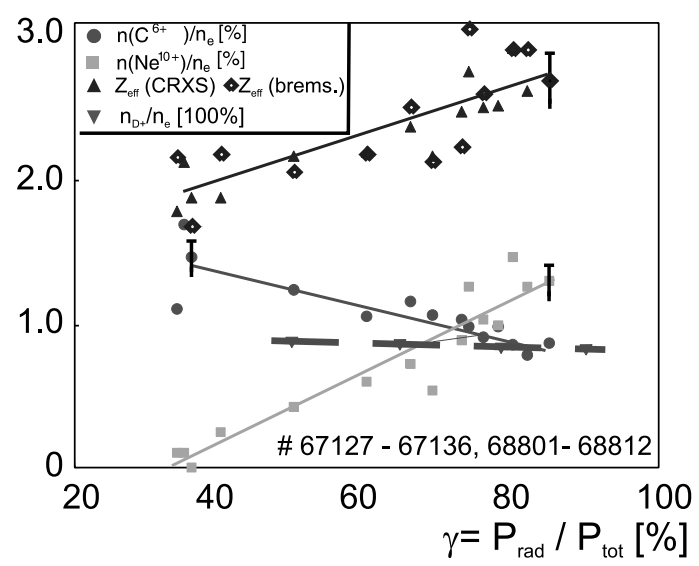

Fig. 3. Central concentration of carbon and neon as measured by CXRS and central deuterium concentration and effective charge as calculated from quasi neutrality and measured from bremsstrahlung against radiation level $\gamma\left(\bar{n}_{e}=0.85-1.0 \cdot n_{G W}, P_{\text {heat }}=2.2-2.7 \mathrm{MW}, I_{p}=\right.$ $400 \mathrm{kA}$; adapted from Ref. 35). 
increasing $\gamma$ despite the constant dilution as carbon is replaced by neon with a higher charge number $Z$. The values of $Z_{\text {eff }}$ deduced from the impurity content measured by CXRS is in agreement with the numbers deduced from a tangential bremsstrahlung measurement along a line of sight through the plasma center. The comparison of the deuterium concentration at the edge and in the center and its variation with increasing $\gamma$ indicates a hollow distribution of the impurity concentration over the plasma radius able to provide a strongly radiating plasma boundary while the plasma reactivity in the center is only weakly affected. This fact is confirmed by a direct measurement of the $Z_{\text {eff }}$ profile based on an Abel inversion of the bremsstrahlung along tangential viewing lines for two RI-mode discharges as shown in Fig. 4a (Ref. 35). The experimental findings could be reproduced with the one-dimensional RITM code ${ }^{37}$ if neoclassical particle drifts only are taken into account together with anomalous diffusion as for the background plasma (see Sec. IV). Of special importance for the resulting hollow concentration profiles is the neo-classical temperature screening at the outer half of the plasma where the impurity ions are in the collisional Pfirsch-Schlüter regime.

As depicted in Fig. 4b, the variation of $Z_{\text {eff }}$ with the plasma density can be fairly well described with the multimachine scaling introduced in Ref. 38, which reads

$$
Z_{\text {eff }}=1+7 P_{\text {rad }} /\left(S \bar{n}_{e}^{2}\right)
$$

and is indicated by the dashed line. Here, $S$ denotes the plasma surface area in square meters, and, $P_{r a d}$ is given in megawatts and $\bar{n}_{e}$ in $10^{20} \mathrm{~m}^{-3}$. The difference between RI-mode discharges with neon or argon seeding and discharges under siliconized wall conditions turned out to be small.

The density of radiated power for a given impurity charge state is mainly determined by the number of excitations per ionization with a maximum for $\mathrm{Li}$ - and $\mathrm{Be}-$ like ionization stages. Therefore, the distribution of the total radiated power is closely linked to the distribution of the most prominent impurity ions up to the Li-like ionization stage. In L-mode discharges without additional impurity seeding this is the intrinsic carbon mainly released from the toroidal ALT-limiter. For carbon the time for ionization up to the Li-like state is too short to establish a poloidally uniform radiating layer (Fig. 5a). Neon, in contrast, leads to formation of a poloidally symmetric radiating belt in the plasma boundary as shown in Fig. 5b. The radial extent of the radiating zone is limited by the radial position where the Li-like charge state is ionized.

Figure 6 shows the comparison between the radial radiation profile in RI-mode discharges with neon and argon, respectively. The electron density and temperature profiles are comparable for both discharges. According to the increase of the ionization potential of the
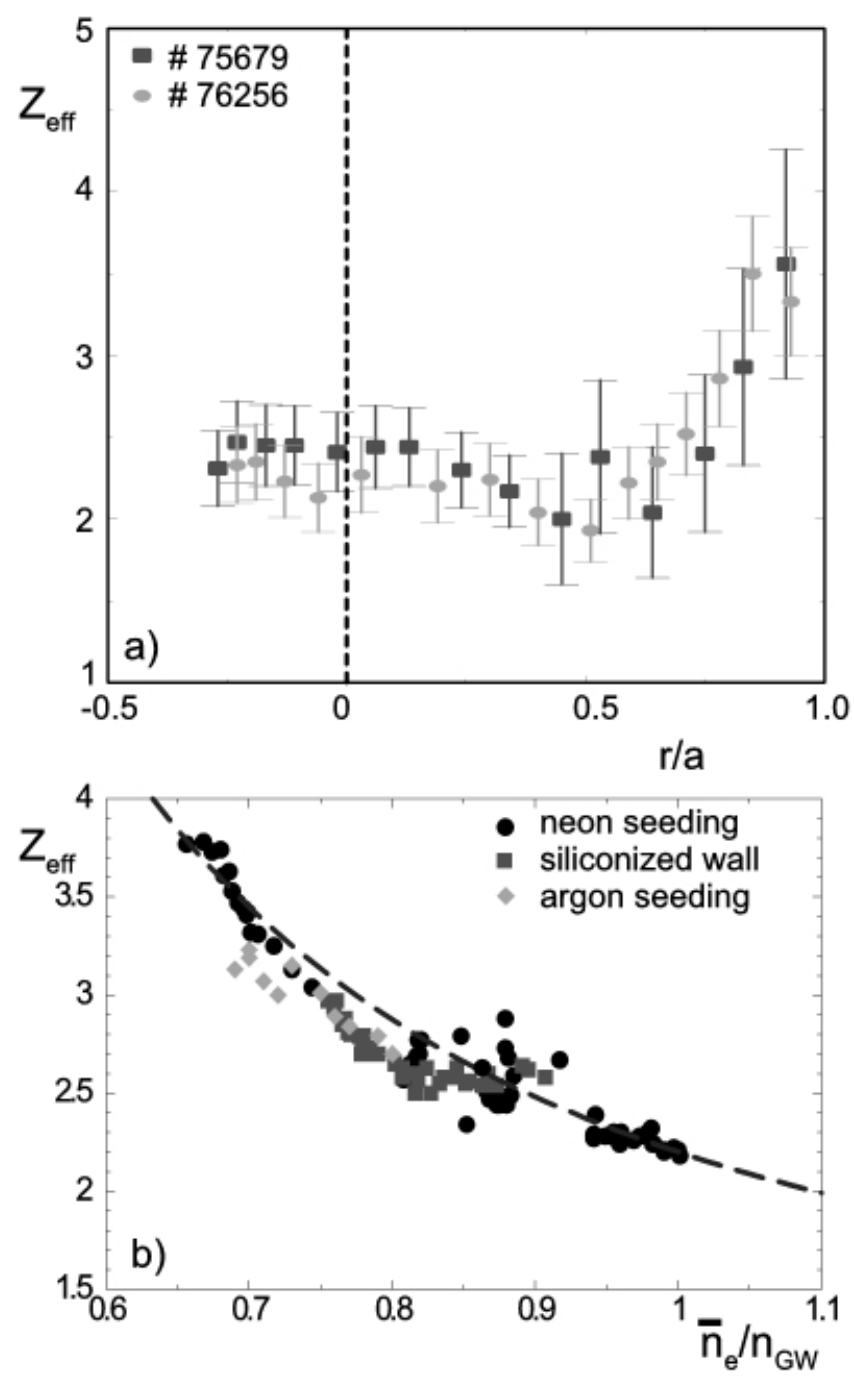

Fig. 4. (a) $Z_{\text {eff }}$ profiles as determined from Abel inversion of tangential bremsstrahlung measurements for two RImode discharges with neon as seeded impurity: one with boronized (\#75679) and one with siliconized wall conditions (\#76256); (b) $Z_{\text {eff }}$ as a function of the density normalized to the Greenwald density for RI-mode discharges with different radiators: circles for neon, squares for silicon, and diamonds for argon. The curve from Eq. (2) is shown by the dashed line, adapted from Ref. 35.

Li-like ionization stage when going from $\mathrm{Ne}$ to $\mathrm{Ar}$ $\left(E_{\mathrm{Li}-\text { like }}^{\mathrm{Ne}}=240 \mathrm{eV}, E_{\mathrm{Li}-\text { like }}^{\mathrm{Ar}}=900 \mathrm{eV}\right)$, we observe a broadening of the radiation zone. In the case of argon the radiating mantle extends up to about half the minor radius. Furthermore, argon is not fully ionized in the plasma core of these discharges at $T_{e}=1300 \mathrm{eV}$. In this respect, argon has a too high charge number for the purpose of radiation cooling in TEXTOR if one sticks to the stringent requirement for the concept of the cold radiating plasma mantle that the line radiation of impurities should be restricted to the plasma boundary. Instead, neon is 


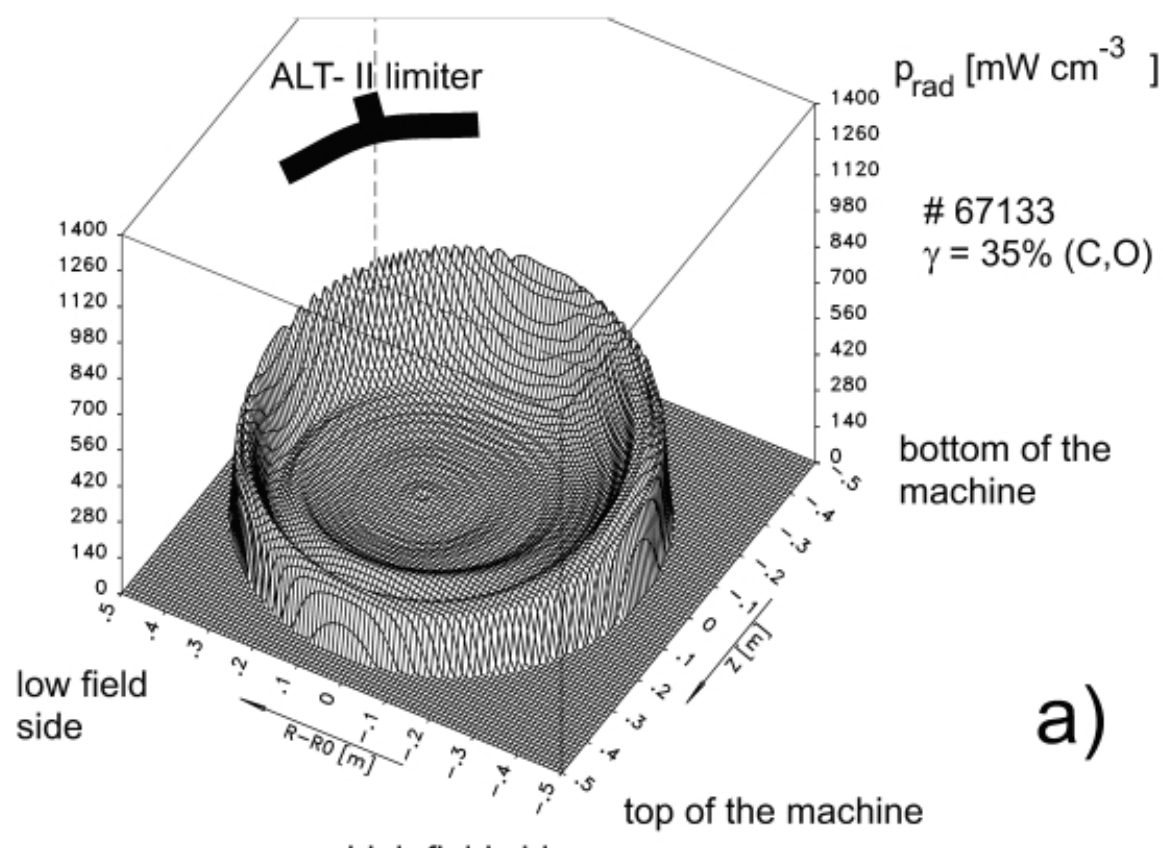

high field side

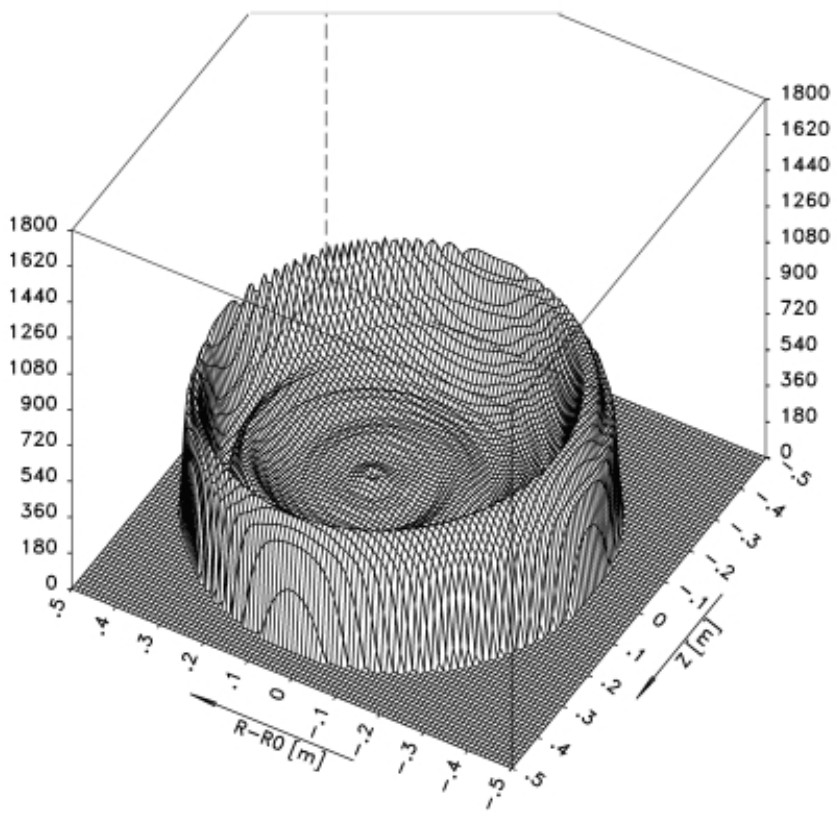

$$
\begin{aligned}
& \mathrm{p}_{\text {rad }}\left[\mathrm{mW} \mathrm{cm}^{-3}\right] \\
& \# 67130 \\
& \gamma=85 \%(\mathrm{Ne}, \mathrm{C}, \mathrm{O})
\end{aligned}
$$

Fig. 5. Tomographic reconstruction of radiated power density $p_{\text {rad }}$ from bolometry in a poloidal cross section of TEXTOR: (a) discharge without additional impurity seeding and (b) discharge with neon seeding $\left(\bar{n}_{e}=5.5 \cdot 10^{19} \mathrm{~m}^{-3}, P_{\text {heat }}=2.5 \mathrm{MW}\right.$, $I_{p}=350 \mathrm{kA}$; adapted from Ref. 17).

optimum with respect to the extent of the radiating mantle. The measured width amounts to about $20 \%$ of the minor radius. In general RI-mode plasmas are shifted toward the low field side by 5 to $20 \mathrm{~mm}$. Under these conditions the onset of MARFEs is avoided even at radiation levels as high as $80 \%$.

\section{GLOBAL CONFINEMENT CHARACTERISTICS IN RI-MODE DISCHARGES IN TEXTOR}

The RI-mode in TEXTOR is obtained with (a) neutral beam coinjection heating possibly combined with neutral beam counterinjection and/or ion cyclotron 


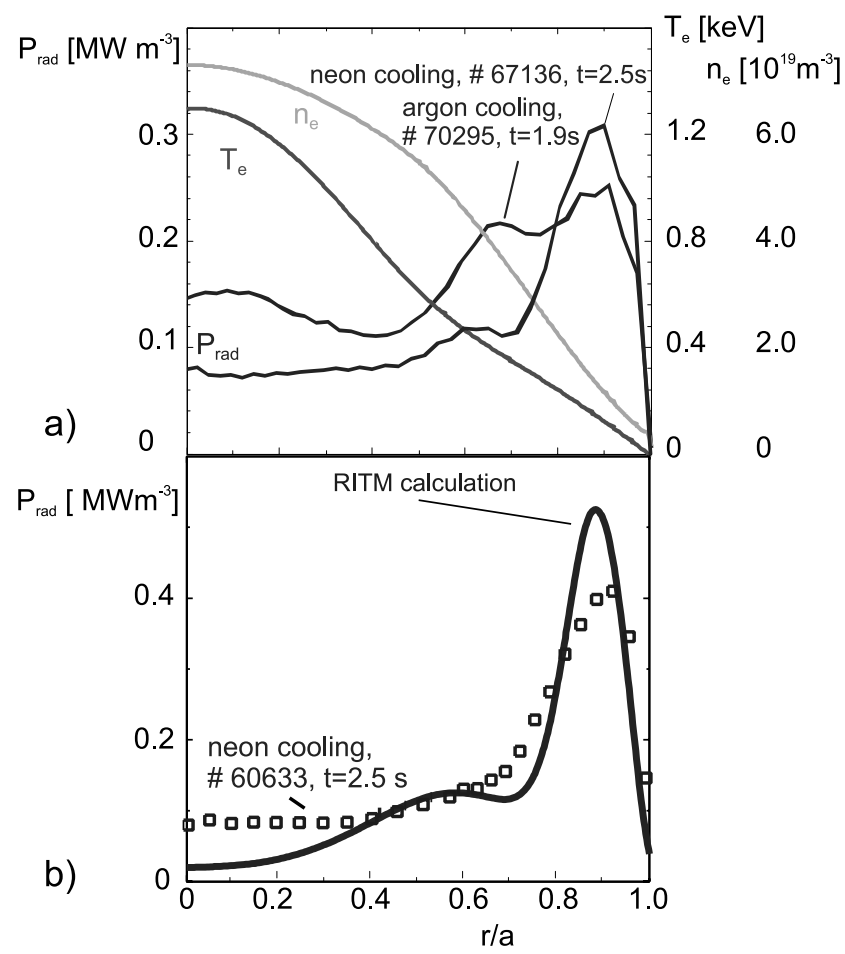

Fig. 6. (a) Radial profiles of the radiated power density for a discharge with neon and argon injection, respectively. Also shown are the profiles of the electron density and temperature $\left(\bar{n}_{e}=5.5 \cdot 10^{19} \mathrm{~m}^{-3}, P_{\text {heat }}=2.5 \mathrm{MW}, I_{p}=\right.$ $400 \mathrm{kA}$; adapted from Ref. 17), (b) Radial profile of the radiated power density in comparison with modeling by the RITM code $\left(\bar{n}_{e}=5.3 \cdot 10^{19} \mathrm{~m}^{-3}, P_{\text {heat }}=2.5 \mathrm{MW}\right.$, $\left.I_{p}=350 \mathrm{kA}\right)$.

resonance heating (ICRH), (b) a feedback controlled seeding of impurities ( $\mathrm{Ne}, \mathrm{Ar})$ in a boronized machine, or (c) sputtering of $\mathrm{Si}$ in a siliconized machine, possibly further enhanced by feedback controlled $\mathrm{Ne}$ seeding. Typical time traces obtained during an RI-mode shot obtained with neutral beam injection (NBI) heating and ICRH on TEXTOR with Ne seeding are given in Fig. 7. In this discharge, the energy content has been kept constant by feedback control of the applied ICRH power. After the start of Ne seeding at $t=1.5 \mathrm{~s}$ (as given by the intensity of a NeVIII line), it takes about $100 \mathrm{~ms}$ before the constant radiation is established as seen from the trace of the radiated power fraction. From that moment, dramatic changes in confinement take place. This can be seen from the strong reduction in the additional heating power by $30 \%$ (ICRH drops from $1.4 \mathrm{MW}$ to about $200 \mathrm{~kW}$ ), without any effect on the stored energy value, and results immediately in a large increase of the H-mode enhancement factor $H 93$, from about 0.83 to 1.0 , corresponding to ELM-free $\mathrm{H}$-mode confinement quality. At the same time, the particle confinement also improves, indicated by the signal for the particle confinement time $\tau_{p}$, and this results in a further increase of the plasma density up to the Greenwald limit.

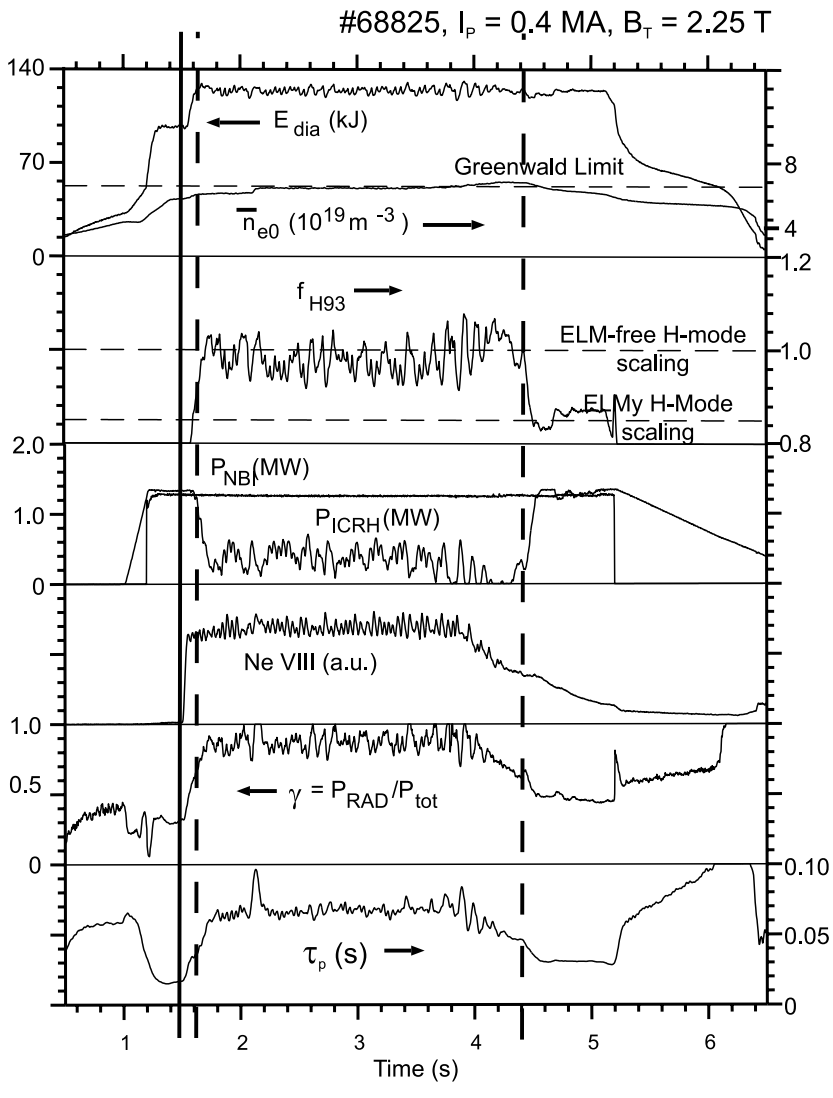

Fig. 7. Time traces for a typical RI-mode discharge: diamagnetic energy and line averaged central electron density, scaling factor of the energy confinement time with respect to the ELM-free H-mode scaling, auxiliary heating power by NBI and ICRH, the intensity of the NeVIII line used for feedback control, the radiated power fraction, and the particle confinement time. The vertical solid line indicates the start of the neon injection, and the dashed lines mark the time interval of the high performance phase.

The energy confinement in the RI-mode is given by the scaling law $\tau_{E, R I}=K \bar{n} P_{t o t}^{-2 / 3}$ (Refs. 15 and 18), where $K=0.18$ if the confinement time $\tau_{E, R I}$ is expressed in seconds, the central line averaged electron density $\bar{n}$ in $10^{20} \mathrm{~m}^{-3}$, and the total heating power $P_{t o t}$ in megawatts. The linear dependence of confinement on the averaged electron density in the RI-mode in TEXTOR is, however, limited by the experimental limit $\beta_{N} \approx 2$ accompanied by the appearance of neoclassical tearing modes ${ }^{39}$ (NTMs). As mentioned already in Sec. II, impurity seeding leads to an increase in the particle confinement time. This has as the consequence that in general TEXTOR RI-mode discharges can maintain a density value close to or substantially above the Greenwald limit by beam fueling only and that a small amount of gas puffing can be sufficient to obtain a large density increase, leading to high values for the energy confinement time $\tau_{E}$ and consequently also for $\beta_{N}$. Therefore, the density increase in this regime is constrained by the maximum $\beta_{N}$. 
The property of the gas fueling to degrade the confinement can be used to advantage by applying a wellchosen moderate level of gas puffing in the discharge, reducing the high confinement of the RI-mode at the highest densities to levels avoiding $\beta$ limitations. The net result is that even higher densities (up to $\bar{n} / n_{G W}=1.4$ ) can now be reached in TEXTOR with ELM-free H-mode confinement quality.

Following the RI-mode scaling given before we can normalize the energy confinement time to compare data at different heating powers and plasma currents. A statistical overview of such normalized confinement times $\tau_{E} P_{\text {tot }}^{2 / 3} / I_{P}$ versus the density normalized to the Greenwald density $\bar{n} / n_{G W}$ is given in Fig. 8 . The data in this diagram belong to a series of shots obtained at $B_{T}=2.25$ $\mathrm{T}$ and $I_{p}$ between 350 and $400 \mathrm{kA}$, with $\mathrm{Si}$ as radiating impurity resulting from sputtering of the siliconized wall of the machine with additional $\mathrm{Ne}$ seeding to obtain higher radiation fractions. Different symbols indicate different values of the neutral pressure in the pumping duct of the toroidal belt limiter ALT-II as a measure of recycling level. The loci of L-, H-, and RI-mode confinement and of the limit $\beta_{N}=2$ are also indicated. Note that RI-mode scaling also describes the Neo-Alcator or linear ohmic confinement (LOC) of TEXTOR (Ref. 20). Points with the lowest fueling rates all follow the RI-mode scaling, which constitutes the best possible confinement that can be achieved in TEXTOR at high density. At higher fueling rates a departure is seen from pure RI-mode confinement, and it is evident from Fig. 8 that by adapting the fueling one can stay just below the $\beta$ limit and nevertheless follow $\mathrm{H}$-mode confinement up to densities

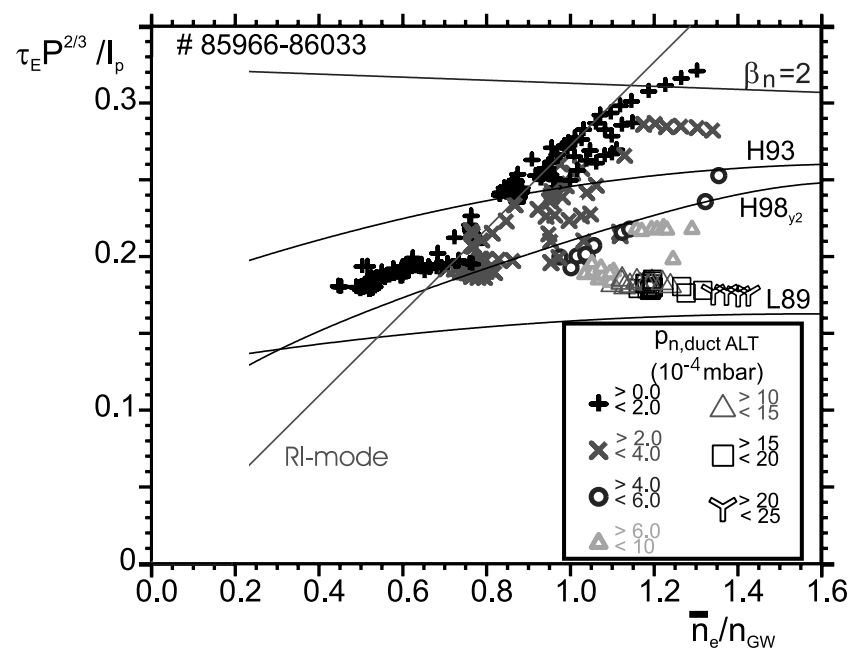

Fig. 8. Overview of energy confinement in the RI-mode in terms of normalized density and energy confinement time in discharges with neon seeding under siliconized wall conditions. Different symbols denote different values of the neutral pressure in the pumping duct of the toroidal belt limiter ALT-II (see text for details). $\bar{n} / n_{G W}=1.4$. The data points belonging to the highest fueling rates all have a confinement close to L-mode.

\section{PHYSICAL MECHANISMS OF THE CONFINEMENT IMPROVEMENT IN THE RI-MODE}

In Sec. II we have already described that the formation of the radiating boundary leads to a lowering of the edge pressure of electrons. Therefore, in order to explain the improved energy confinement and the higher energy content in the RI-mode as seen, e.g., in Fig. 7, we expect a zone of steeper pressure gradients further inside the plasma bulk. Figure 9 shows that such a steepening of the pressure profiles (here depicted in the electron channel) can indeed be observed around $r / a=0.6$, where the radiating plasma mantle - as shown by the radial profile of the radiated power density-ends. The electron pressure gradient length $L_{p}=p /(\partial p / \partial r)$ decreases by a factor of 2 at its maximum around $r / a=0.4$.

The increase of the central pressure is mainly caused by a characteristic steepening of the density profile in the RI-mode as shown in Fig. 10. The core temperature profiles of electrons and ions are closely coupled at the high densities, and both channels show a slight increase of the central temperatures in the RI-mode. Furthermore, for the pair of discharges shown in Fig. 10, which were heated by neutral beam coinjection, we observe an increase of the central toroidal rotation indicating an improvement of the confinement for angular momentum similar to the improvement of energy confinement.

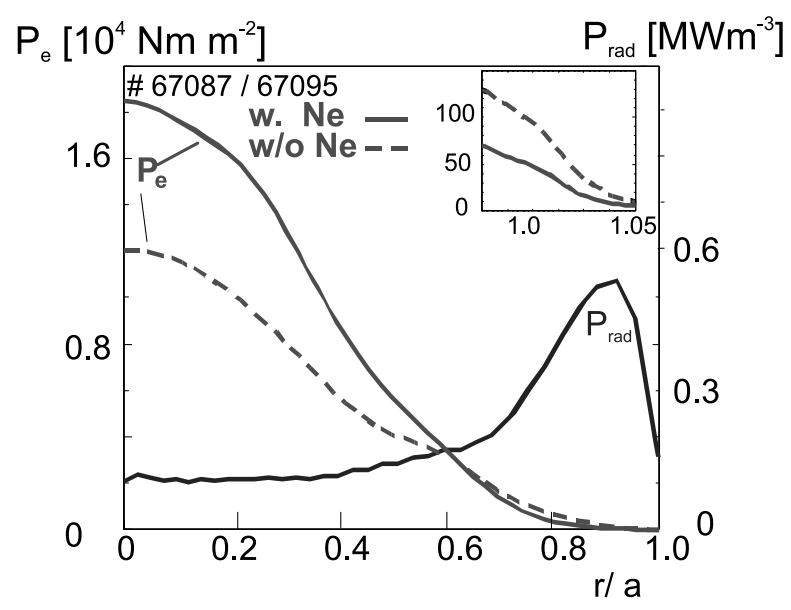

Fig. 9. Radial profile of the radiated power density and the electron pressure for an RI-mode discharge with neon injection and an L-mode discharge (dashed lines) without neon injection $\left(\# 67086 / 67095, t=2.5 \mathrm{~s}, \bar{n}_{e}=\right.$ $\left.5.2 \cdot 10^{19} \mathrm{~m}^{-3}, P_{\text {heat }}=2.5 \mathrm{MW}, I_{p}=350 \mathrm{kA}\right)$. Insert shows a magnification of the edge pressure profiles (adapted from Ref. 17). 


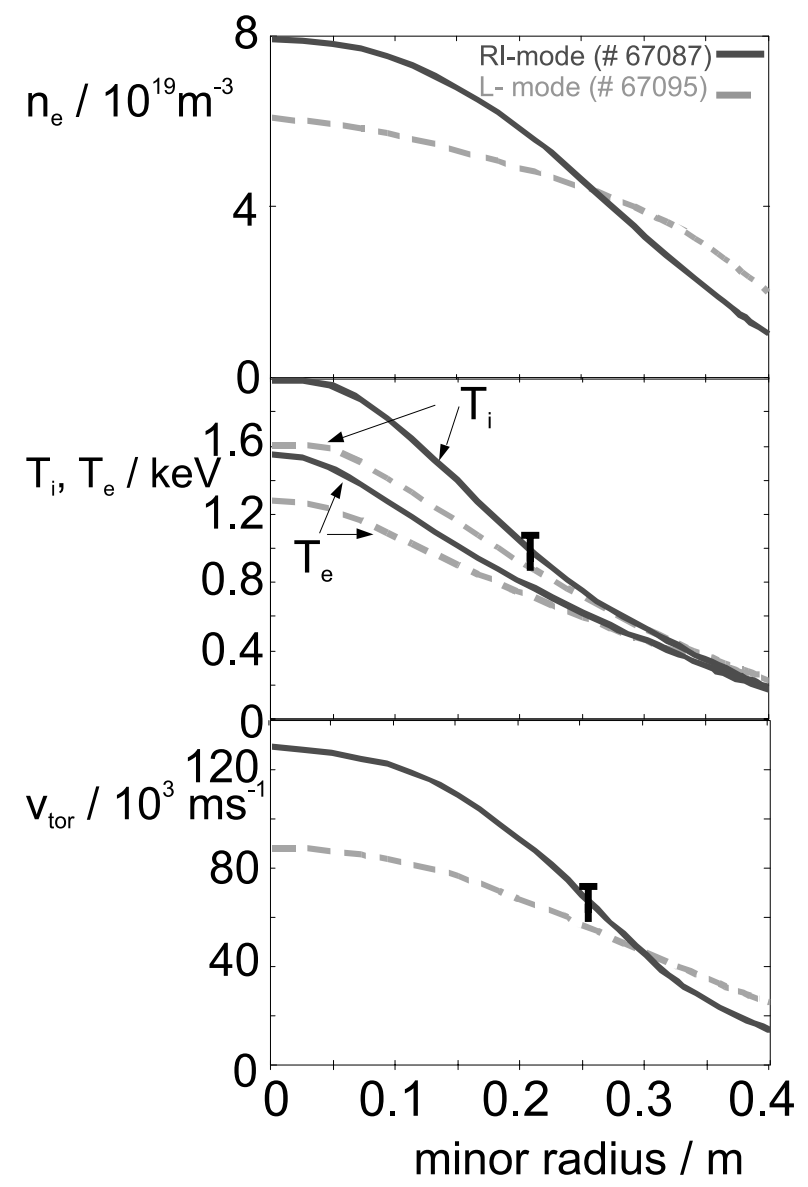

Fig. 10. Radial profile of the electron density, ion and electron temperature, and toroidal velocity in an RI-mode discharge and an L-mode discharge (\#67086/67095, $t=2.5 \mathrm{~s}, \bar{n}_{e}=5.2 \cdot 10^{19} \mathrm{~m}^{-3}, P_{\text {heat }}=2.5 \mathrm{MW}, I_{p}=$ $350 \mathrm{kA})$.

The strong correlation between the steepening of the density profile and the global energy confinement observed experimentally points to a suppression of microturbulence due to ion temperature gradient (ITG)-driven modes as the cause for the improvement during L-RI transitions. We recall that an important parameter to characterize ITG-driven transport is the ratio between the scale lengths of density and temperature profiles of plasma ions defined as $\eta_{i}=L_{n} / L_{T_{i}}$. Therefore, a steepening of the density profiles leading to a decrease of $L_{n}$ may point to lower $\eta_{i}$ and increased stability against ITG modes and smaller transport.

Evidence for a suppression of ITG-driven turbulence has first been found in analyzing the experimental profiles shown in Fig. 10 and calculating the growth rate spectra. ${ }^{40}$ Here, the effect of the impurities has explicitly been taken into account. Figure 11 shows the resulting growth rate spectra of the toroidal ITG (no consideration of the slab ITG branch) for the L- and RI-mode profiles of Fig. 10 at $r / a=\frac{2}{3}$. This multifluid calculation has been

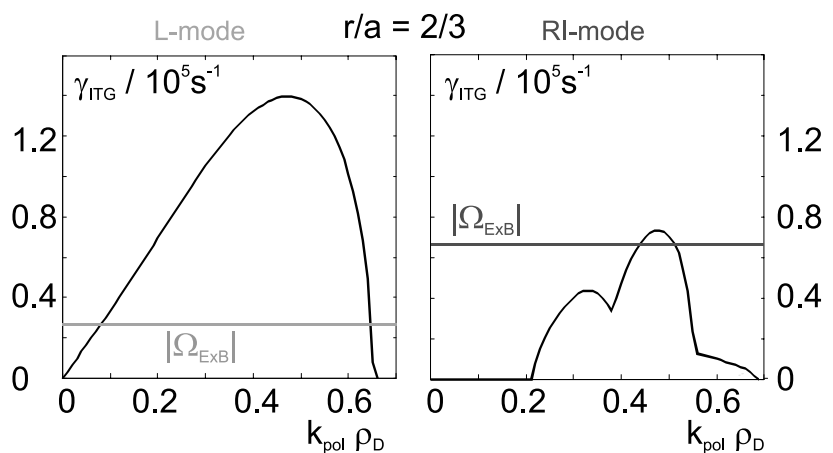

Fig. 11. Growth rate spectra of the ITG mode, multifluid calculation using the density profiles of deuterium and $\mathrm{C}^{6+}$ for the L-stage and deuterium, $\mathrm{C}^{6+}, \mathrm{Ne}^{10+}, \mathrm{Ne}^{9+}$, and $\mathrm{Ne}^{8+}$ for the RI-stage, respectively. The local $E \times B$ shearing rate is shown for comparison.

performed taking into account the $\mathrm{C}^{6+}$ profiles from CXRS for the L-stage and $\mathrm{C}^{6+}, \mathrm{Ne}^{10+}$ (both from CXRS), $\mathrm{Ne}^{9+}$, and $\mathrm{Ne}^{8+}$ (from RITM modeling) for the RI-stage. The deuteron profiles have been deduced assuming quasi neutrality. We observe a significant reduction of the growth rate in the RI-mode and important effects related to the presence of the impurities. Furthermore, the growth rate is reduced to zero for small $k_{p o l} \rho_{H}$ during the RI-stage.

Additionally, for both L- and RI-stage the local $E \times B$ shearing rate $\Omega_{E \times B}$ is plotted, which shows the significant further reduction of the effective growth rate $\gamma_{I T G}-$ $\Omega_{E \times B}$ for this discharge where a substantial toroidal torque has been exerted onto the plasma by the neutral beam coinjection.

The suppression of the ITG mode in the RI-stage as found from the multi-fluid analysis has been confirmed in three-dimensional non-linear gyrokinetic particle-incell simulations in toroidal geometry including multiple ion species. ${ }^{22,41,42}$

All the calculations show that the peaking of the density profile has the strongest impact on the ITG mode. With respect to the dynamics of the bifurcation, however, we have to consider the direct influence of the impurities on the core confinement. We can expect a beneficial impact of impurities on the growth rate of the ITG mode if we consider a simple averaged ion approximation where the dependence of the threshold of the ITG mode $\eta_{i, c r i t}$ on the effective plasma charge $Z_{\text {eff }}$ is given as ${ }^{43}$

$$
\eta_{i, \text { crit }}=\frac{2}{3}+\varepsilon_{n}\left(\frac{10}{9 Z_{\text {eff }}}+\frac{Z_{\text {eff }}}{4}\right)+\frac{Z_{\text {eff }}}{4 \varepsilon_{n}}-\frac{Z_{\text {eff }}}{2} .
$$

Here, $\varepsilon_{n}=2 L_{n} / R$ relates the density gradient length to the gradient length of the toroidal magnetic field, and we assume equal ion and electron temperatures. A simple expression of the growth rate of the toroidal ITG mode can then be given as 


$$
\gamma_{I T G}=\frac{\omega_{D e}}{Z_{e f f} \varepsilon_{n}^{1 / 2}} \sqrt{\eta_{i}-\eta_{i, c r i t}},
$$

where $\omega_{D e}=2 k_{p o l} T_{e} / e B R$ denotes the magnetic drift frequency.

For typical density profiles under L-mode conditions in TEXTOR we observe $\varepsilon_{n}=0.3-0.4$ around half the minor radius. Under these conditions, an increase of $Z_{\text {eff }}$ stabilizes ITG modes. The important question now is how an initial reduction of the ITG-driven transport can cause the characteristic steepening of the density profile, which then amplifies the turbulence suppression owing to the dependence of the ITG threshold on the gradient length of the density profile $L_{n}$. A steepening of the density profile could be attributed to the presence of additional particle sources. However, a modeling of the source distribution shows that the strong peaking of the density profile deep in the plasma bulk cannot be attributed solely to changes of the source distribution. Therefore, we have to look for a change of an increase of the ratio between inward particle pinch and outward particle diffusion.

The interplay between the direct influence of the impurities on the ITG mode and the subsequent evolution of the profiles has been first explained in Ref. 44 based on a transport model based on ITG and dissipative trapped electron (DTE) modes. For the growth rate of the DTE mode we use the expression given in Ref. 45:

$$
\gamma_{D T E M}=f_{t r} \eta_{e} \omega_{*} \frac{\omega_{*} \nu_{e, e f f}}{\omega_{*}^{2}+\nu_{e, e f f}^{2}},
$$

where $f_{t r}$ denotes the fraction of trapped particles, $\eta_{e}=$ $L_{n e} / L_{T e}$ is the ratio of the characteristic gradient lengths of the electron density and temperature profile, $\omega_{*}=$ $-T_{e} k_{\perp} /\left(e B L_{n}\right)$ is the diamagnetic drift frequency with the perpendicular wave vector chosen as the inverse of the ion Larmor radius, and $\nu_{e, \text { eff }}$ is the effective electron collision frequency.

While for the particle flux driven by the ITG mode the particle pinch is relatively weak and neglected, henceforth $\left[\Gamma_{I T G} \simeq-D_{I T G}(\partial n / \partial r)\right]$, the DTE mode is associated with a particle pinch related to the profile of the safety factor $\left[\Gamma_{D T E} \simeq-D_{D T E}(\partial n / \partial r)+(n \partial q / \partial r)\right]$. Here, the diffusion coefficients are estimated in a mixing length approximation $D \sim \gamma / k_{\text {pol }}^{2}$.

The role of the effective plasma charge $Z_{\text {eff }}$ as a control parameter for the L-RI transition can be easily illustrated if we take a fixed $L_{T}$ and consider only the particle balance equation neglecting particle sources and temporal variations $\left(\Gamma_{I T G}+\Gamma_{D T E}=0\right)$. We define a peaking factor for the density profile with respect to the temperature profile $p=L_{T} / L_{n}$, which is just the inverse of the $\eta_{i}$ parameter usually discussed in context with the ITG mode. Under these assumptions the particle balance equation can be written as ${ }^{44}$

$$
\begin{aligned}
\Gamma\left(p, Z_{\text {eff }}\right)= & \frac{1}{0.4} \sqrt{\frac{L_{T}}{p Z_{\text {eff }}}\left[\frac{1}{p}-\frac{1}{p_{\text {crit }}}\right]} \cdot p \\
& +\frac{\eta^{*}}{p^{2}+\eta^{*^{2}}} \cdot\left(p-p_{q}\right)=0 .
\end{aligned}
$$

The first part of the sum in Eq. (6) corresponds to the transport based on the ITG mode (the factor in front of the square root expression stems from our choice of the wave vector $\left.k_{p o l}=0.4 / \rho_{s}\right)$. The ITG threshold is defined as $p_{\text {crit }}=1 / \eta_{\text {crit }}$ with $\eta_{\text {crit }}$ given in Eq. (3). The second part of the sum describes the transport owing to the DTE mode with the dimensionless factor $\eta^{*}=$ $L_{T} \nu_{\text {eff }} / \omega^{*}$, which is proportional to the ratio between the collision frequency $\nu_{\text {eff }}$ and the drift frequency $\omega^{*}$, which both enter the DTE growth rate. Furthermore, we have introduced $p_{q}=L_{T} / L_{q}$ as the normalized peaking factor for the profile of the safety factor. Figure 12a shows the influence of the peaking factor $p$ on the particle flux for three different values of $Z_{\text {eff }}$ corresponding to the L-mode $\left(Z_{\text {eff }}=1.5\right)$, to the transition to the RI-mode $\left(Z_{\text {eff }}=2.15\right)$, and to the developed RI-mode state $\left(Z_{\text {eff }}=2.5\right)$ for typical plasma parameters in RImode discharges at $r / a=0.6$ in TEXTOR, $\eta^{*}=0.7$, $L_{T} / R=0.08$, and $p_{q}=0.8$. The solution of Eq. (6) as a function of both $p$ and $Z_{\text {eff }}$ is depicted in Fig. 12b. Two different branches can be distinguished, one branch governed by the ITG mode, which is corresponding to the L-mode stage, and one branch characterized by $L_{n} \sim$ $L_{q}$, where the ITG mode is suppressed and transport is governed by the DTE mode. This stage corresponds to the RI-mode. We clearly note the existence of a critical value for $Z_{\text {eff }}$ to obtain a bifurcation to the $\mathrm{L}$ - to the RI-stage where the ITG mode is suppressed $\left(Z_{\text {eff }}=\right.$ 2.15 under these conditions). It has been shown in Ref. 44 that such a model can successfully describe the dynamic evolution of the density profile observed experimentally and that without the increase of $Z_{\text {eff }}$ the transition to the RI-mode cannot be obtained. The model discussed here does not include a possible bifurcation of the toroidal velocity shear because of $E \times B$ stabilization of the ITG mode. Such a possibility has been discussed in Ref. 46 for the case of strong momentum input in discharges with unbalanced neutral beam heating. The importance of $E \times B$ shear to amplify the effect of impurities on ITG modes has also been emphasized in Refs. 24, 25, and 26 for impurity seeded discharges in DIII-D as discussed in Sec. V.

Finally, we point to the fact that within this model the linear dependence of the confinement on the density in the RI-mode in TEXTOR can be explained by the decrease of the DTE growth rate $\gamma_{D T E M} \propto 1 / n_{e}$ with density for the plasmas in TEXTOR with rather high collisionality $\left(\omega_{*} \ll \nu_{e, e f f}\right)$. 

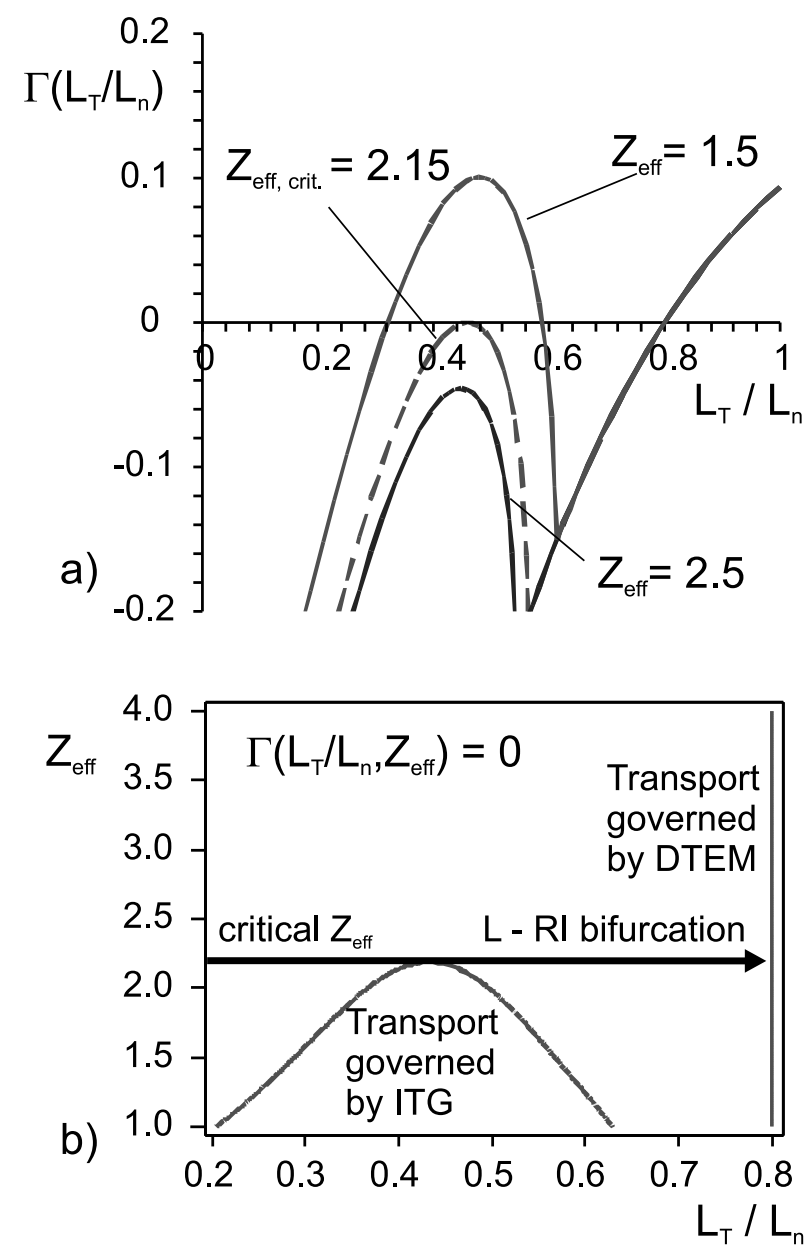

Fig. 12. (a) Normalized particle flux as used in the particle balance Eq. (6) as a function of the normalized peaking factor for the density profile $p=L_{T} / L_{n}$ for different effective plasma charges $Z_{\text {eff }}$ (corresponding to L-mode, L-RI transition, and RI-mode) and (b) solution of the balance equation as a function of $p=L_{T} / L_{n}$ and the effective plasma charge $Z_{\text {eff }}\left(p_{q}=0.8\right.$, $L_{T} / R=0.08, \eta^{*}=0.7$; see text for details).

\section{EXTRAPOLATION TO LARGER DEVICES}

The results obtained on TEXTOR as discussed in the previous sections show the potential of impurity seeding as a technique to mitigate heat loads to the edge, compatible with keeping high density and good confinement. The question arises in how far the TEXTOR results will be applicable to ITER. To this end, experiments have been undertaken on a large number of machines throughout the world, and in what follows, we will briefly summarize the main results obtained on divertor machines up to now. It is important to note that these experiments did not show the characteristic density peaking as observed in the RI-mode at TEXTOR. Nevertheless, a beneficial impact of impurities on transport is seen under many circumstances (e.g., with the help of $E \times B$ shear as mentioned before). In the following these regimes are called radiative modes to distinguish the scenarios from the RI-mode.

\section{V.A. Radiative Mantle Experiments in DIII-D}

Various configurations have been tested on this machine ${ }^{24-26}$; one of them in lower single null is called "puff and pump" ELMy H-mode discharge (where a large deuterium gas flow is applied together with the strike points being positioned near the entrance of the divertor cryopumps for particle removal ${ }^{26}$ ). All discharges have been obtained with neutral beam coinjection as the only additional heating source.

Argon seeding in puff and pump ELMy H-mode discharges led to a decrease of the edge pedestal pressure by about $33 \%$ with increasing radiating power fraction from 0.4 to 0.8 (Ref. 26) for a nearly constant enhancement factor $H 98(y, 2)$. Impurity seeded puff and pump ELMy $\mathrm{H}$-mode discharges allow operation at higher densities compared to unseeded reference discharges without degradation of confinement.

In diverted L-mode discharges with neon seeding, a reduction has been observed in the effective thermal and toroidal momentum diffusivity with a concomitant increase in the thermal neutron production. ${ }^{26}$ An increase in toroidal rotation and in the $E \times B$ shearing rate has been observed, contributing to the stabilization of microturbulence, and may be part of the explanation for the decrease in effective thermal diffusivity. Experimentally one finds a reduction of ITG turbulence to be at work in these discharges, measured by beam emission and far infrared spectroscopy. ${ }^{47}$ Inner wall limited L-mode discharges with neon seeding exhibit behavior qualitatively similar to RI-mode discharges in TEXTOR as an increase of the particle confinement and a rise of the $\mathrm{H}$-factor with density. ${ }^{26}$

\section{V.B. Radiating Mantle Experiments in JET}

Radiative mantle plasmas in ELMy H-mode discharges have been realized on JET as the result of an intensive campaign over the last years. Two configurations have emerged as particularly promising: the ELMy $\mathrm{H}$-mode discharges with the X-point on the septum in the Gas Box divertor MkII-GB and the ELMy H-mode in the recently modified JET MkII-SRP divertor where the septum is removed and substituted with a septum replacement plate.

\section{V.B.1. Impurity Seeding in Low Triangularity ELMy $H$-Mode Discharges in JET with X-Point on the Septum}

This configuration ${ }^{30,31}$ resembles a combination of a divertor and limiter configuration. Because of the lower $\mathrm{H}$-mode power threshold, these plasmas have the added 
advantage to stay in $\mathrm{H}$-mode even at high radiation levels. Figure 13 (Ref. 30) illustrates a typical time sequence for such a discharge. After the start of the additional heating, a strong gas puff is applied to increase the density. Confinement is strongly reduced in this phase due to the strong gas puffing as also observed on TEXTOR and in other plasma operational scenarios on JET. The confinement degradation is clearly shown in the reduction of the enhancement factor $H 98(y, 2)$ decreasing from 1.13 to 0.76 . During the $D_{2}$ puffing phase, additional Ar seeding leads to an increase in the radiative power level and in the plasma density as a result of an increase in the particle confinement time during impurity seeding, as observed on TEXTOR. After the closure of the $D_{2}$ gas fueling valve, the density remains the same, but confinement is restored, and in this way we reach outstanding values for the product $H 98(y, 2) \cdot \bar{n} / n_{G W} \sim 1.0$ at $\bar{n} / n_{G W} \sim$ 0.9 and radiation fractions $P_{\text {rad }} / P_{\text {tot }}$ up to $60 \%$. Those values can last for $5 \mathrm{~s}$ equivalent with 12 confinement times. This phase is called in what follows the "afterpuff" phase. During this afterpuff phase, careful Ar and $D_{2}$ puffing is applied to keep the radiation level and the plasma density as constant as possible. For the dis-

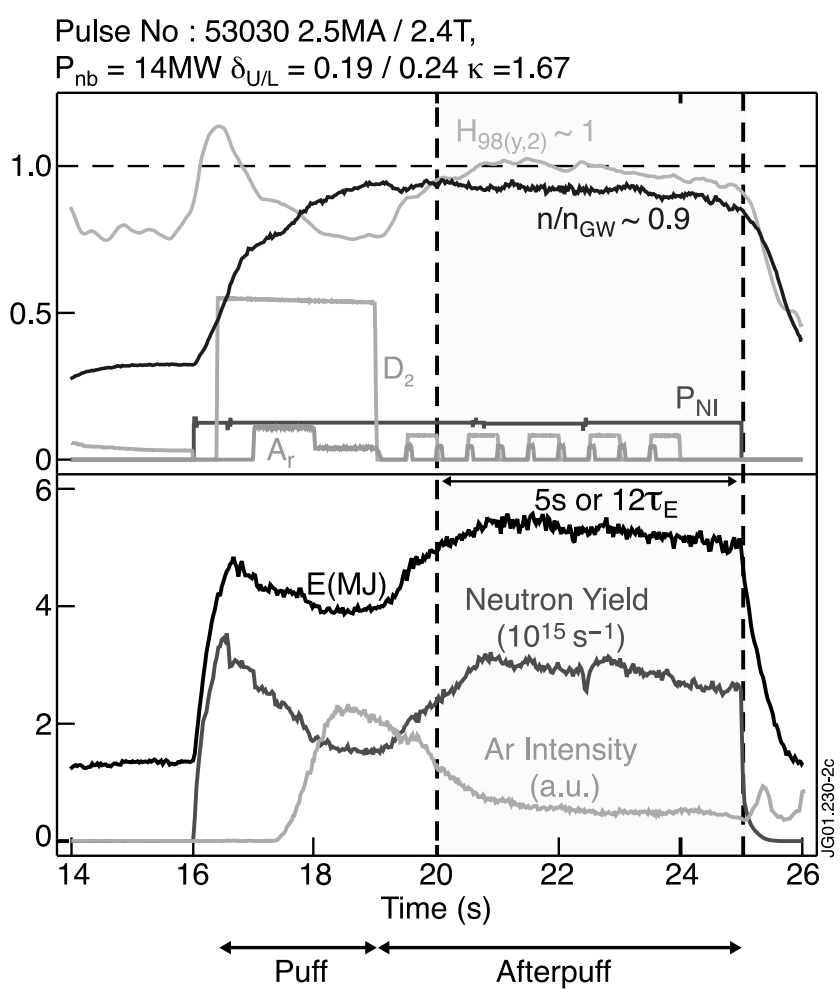

Fig. 13. Time traces of the H-mode confinement factor $H 98(y, 2)$, the Greenwald factor, the neutron yield, the diamagnetic energy, argon line emission from the core, and the timing of the external deuterium and argon injection. ${ }^{30}$ charges under consideration most of the radiation is localized in the edge and not in the divertor and/or X-point, typically $P_{\text {divertor }} / P_{\text {tot }} \sim 15$ to $20 \%$ for impurity seeded ELMy H-mode discharges in the septum configuration. Argon seeding is more adapted to the JET plasma parameters than $\mathrm{Ne}$, as the latter leads for a given radiation level to a higher $Z_{\text {eff }}$.

\section{V.B.2. Impurity Seeding in High Triangularity ITER-Like Plasmas}

Argon seeded ITER-like plasmas ${ }^{30-32}$ (EHT configuration, 2.3 MA/2.4 T) showed a radiating mantle and $P_{\text {rad }} / P_{\text {tot }}=60$ to $70 \%$, illustrated in Fig. 14b (Ref. 30), where Ar seeding starts at $t=20 \mathrm{~s}$. ICRH has been applied to avoid impurity accumulation. After the establishment of the radiation (which takes about $500 \mathrm{~ms}$, as can be seen from the signal for $\left.P_{\text {rad }} / P_{t o t}\right)$, Argon seeding correlates with an increase in the plasma density, up to $1.2 n_{G W}$, for a constant value of the enhancement factor $H 98(y, 2) \sim 1$, which is the same value as the unseeded counterpart (Fig. 14a). $Z_{\text {eff }}$ is somewhat increased from $\sim 1.7$ to $\sim 2$.0. Infrared measurements at a fixed location in the inner and outer divertor showed a reduction in the surface temperature of the divertor target with Ar seeding. The effect is clearly visible in the inner divertor, and even more in the outer divertor, where the base level is dropping from about $500^{\circ} \mathrm{C}$ to about $100^{\circ} \mathrm{C}$ and also the maximum temperature spikes due to ELMs are reduced from $1300^{\circ} \mathrm{C}$ to $600^{\circ} \mathrm{C}$. These results have been confirmed in subsequent experiments by measurements of thermocouples in the divertor target tiles. Further optimization of these discharges has been obtained at 2.5 MA/2.7 $\mathrm{T}$ in the so-called HT3 configuration on JET (which is a slightly adapted EHT configuration, in view of future operation at higher plasma currents) by applying a simultaneous feedback control of both the $H 98(y, 2)$ enhancement factor and the radiation fraction. Stationary values for the radiated power fraction, plasma beta, density stored energy, and neutron emission have been obtained in this way for the full duration of the feedback phase $\left(5 \mathrm{~s}\right.$ or $12 \tau_{E}$ ), which is the maximum duration for such discharges on JET. Under these conditions the ITG growth rate can be reduced by a factor of 2 at half the minor radius with argon seeding in comparison with an unseeded reference. ${ }^{32}$

The presence of the impurities also changes the character of the ELMs. Impurity seeding (by Ar or Ne) applied during the main $D_{2}$ puffing phase can change the ELMs from type I to more benign high frequency type III ELMs (see also Ref. 48 for a detailed study on type III ELMy H-mode discharges with nitrogen seeding). Depending on the conditions mixed type I/II ELM phases also have been observed. Impurity seeding thus provides an additional knob to change the behavior of the ELMs and thus possibly is a means to optimize in a general way the properties of the H-mode. 

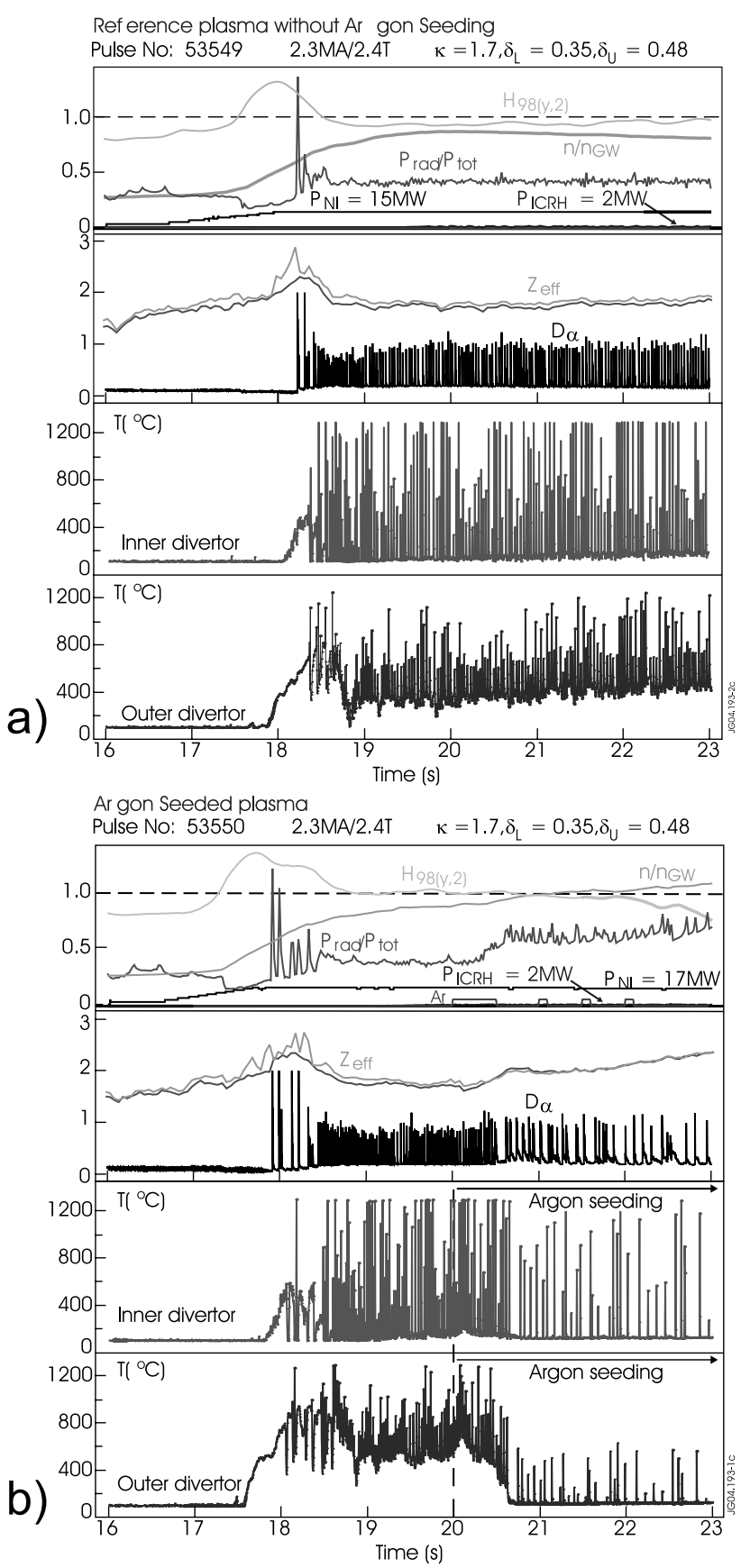

Fig. 14. Time traces of the H-mode confinement factor $H 98(y, 2)$, the Greenwald factor, the radiated power fraction $P_{\text {rad }} / P_{t o t}$, the heating power, the effective charge number $Z_{\text {eff }}$, the Balmer- $\alpha$ emission in the divertor, and the target temperature in the outer and inner divertor for (a) a no Ar reference ELMy H-mode discharge and (b) a discharge with Ar injection, both at high triangularity. ${ }^{30}$

\section{V.C. Impact of Impurities on Transport in Large Machines}

At the end of this review we want to come back to the question of how the seeded impurities influence the trans- port properties in discharges with a radiating plasma mantle in large machines with the aim to give an outlook to future devices like ITER. We will show that the experimental findings, especially in JET, can be explained by the simple model introduced in Sec. IV to a large extent.

An important point is the access to peaked density profiles, which is the characteristic property in RI-mode discharges in TEXTOR. In ELMy H-mode discharges in JET (Ref. 31) as well as in DIII-D (Ref. 26) such a bifurcation has not been observed. The energy confinement in general does not deteriorate (if the H-mode pedestal is not reduced to much and type I ELMs can be retained), but no confinement transition is found as a consequence of the impurity seeding. In L-mode discharges at low densities, however, a confinement improvement has been found in both JET (Ref. 49) and DIII-D (Refs. 26 and 47), albeit at a much higher $Z_{\text {eff }}$ as needed for the L-RI transition in TEXTOR.

At first, the absence of a linear scaling of the energy confinement time with density as observed in TEXTOR can be related to the lower collisionality in these devices, which results in a different dependence of the DTEM growth rate on density for $\omega^{*} \gg \nu_{e, e f f}[$ see Eq. (5)]. To discuss the difference between $\mathrm{H}$-mode and L-mode discharges and the need for higher $Z_{\text {eff }}$ at lower collisionalities we use again Eq. (6) to calculate the critical $Z_{\text {eff }}$ for a suppression of the ITG mode and a bifurcation to peaked density profiles. Again we assume no particle sources and fix the temperature gradient length $\left(L_{T} / R=0.12\right.$ in the following).

Figure 15a shows $Z_{\text {eff }}$ as a function of the normalized collisionality parameter $\eta^{*}=L_{T} \nu_{\text {eff }} / \omega^{*}$ for a fixed peaking factor of the $q$ profile, $L_{T} / L_{q}=1.0$; in Fig. $15 \mathrm{~b}$ we have varied $L_{T} / L_{q}$ for $\eta^{*}=0.7$. At first the model suggests an increase of the critical $Z_{\text {eff }}$ for low collisionalities while it is minimum for typical TEXTOR values $\eta^{*}=0.7$. However, we have to keep in mind that the model does not include the collisionless trapped electron mode. Therefore, the extrapolation to lower collisionalities remains uncertain although our findings provide an explanation for the need for higher $Z_{\text {eff }}$ in low density L-mode discharges at JET.

Moreover, there is a dramatic increase of the critical $Z_{\text {eff }}$ for flatter $q$ profiles. This finding can qualitatively explain the very flat density profiles generally observed in H-mode plasmas and the difficulties to observe a transition to a peaked density stage when impurities are seeded in ELMy H-mode plasmas. The $\mathrm{H}$-mode is characterized by an edge pedestal in the temperature owing to the resulting low collisionality in the pedestal the edge current density is relatively larger than in L-mode plasmas. As a consequence the internal inductance (as a measure of the peakedness of the current density profile) is small. The bootstrap current developing at the edge owing to the pressure pedestal amplifies this fact. These conclusions based on a simple analysis of the particle balance have been confirmed with extensive transport modeling 
a)
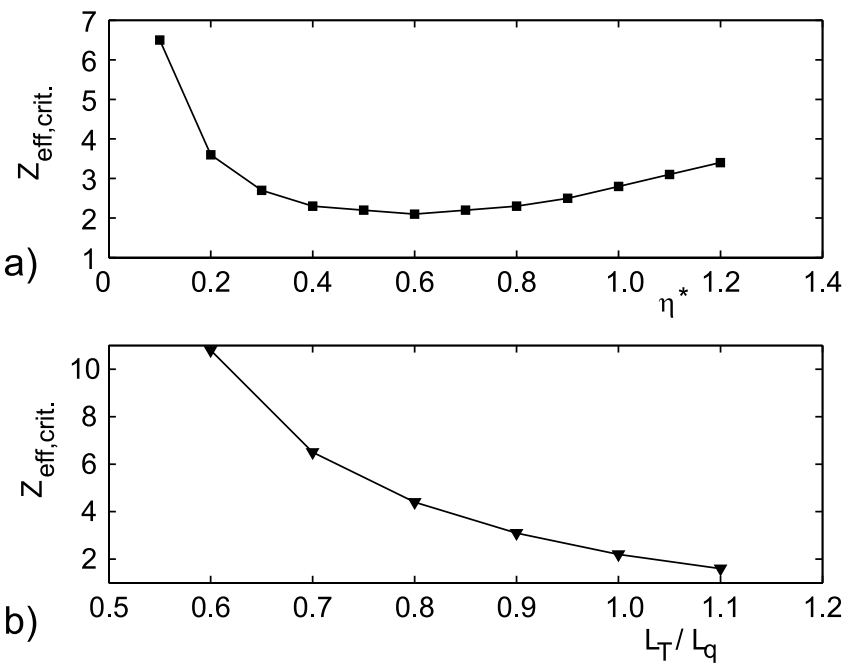

Fig. 15. Critical $Z_{\text {eff }}$ to obtain a bifurcation to peaked density profiles via the suppression of ITG modes based on the particle balance equation (no sources, $L_{T} / R=$ 0.12 ); (a) as a function of the normalized collisionality $\eta^{*}=L_{T} \nu_{\text {eff }} / \omega^{*}$ for $L_{T} / L_{q}=1$ and (b) as a function of the normalized peaking factor of the $q$-profile $L_{T} / L_{q}$ for $\eta^{*}=0.7$ (see text for details).

of neon seeded L-mode discharges ${ }^{50}$ and argon seeded $\mathrm{H}$-mode discharges ${ }^{51}$ with the RITM code. Currently, a model for the impact of impurities on transport is being developed that is applicable to arbitrary collisionalities following the prescription given in Ref. 45. This model takes into account that ITG and trapped electron modes cannot be separated in general. Initial calculations show that the picture is not significantly changed for typical RI conditions in TEXTOR (Ref. 52). The dominant influence of the $q$ profile on the possibility to obtain a bifurcation to a peaked density profile persists as the particle pinch related to trapped electron modes is still linked to the $q$ profile as discussed in Sec. IV.

\section{SUMMARY AND CONCLUSIONS}

Extensive studies on plasmas with a cold radiating mantle have been performed in the tokamak TEXTOR. It has been shown that up to $90 \%$ of the total input power can be radiated under quasi-stationary conditions with reactor-relevant heat flux densities at plasma densities around the empirical Greenwald density. A transition to a state with improved confinement triggered by the impurity injection and accompanied by a significant peaking of the density profile has been found, the RI-mode. Detailed studies on the physical mechanisms responsible for the confinement improvement have been made, including numerical modeling based on drift-wave-based anomalous transport and self-consistent treatment of im- purities and background plasma. As a result, a suppression of ITG modes has been found as the most probable cause for the confinement improvement. Based on the experience in TEXTOR and in view of the application of impurity seeding to future devices like ITER to mitigate the problem of power exhaust, experiments with radiative modes on large devices like JET have been conducted.

Based on the experience gained in $\mathrm{H}$-mode discharges in JET and supported by our modeling, we do not expect the possibility to trigger a bifurcation to peaked density profiles in the bulk of an $\mathrm{H}$-mode plasma in ITER. Also, the absence of strong toroidal momentum input limits the prospect for a bifurcation to improved confinement via an increase of $E \times B$ shear. More detailed modeling is needed for a better extrapolation to conditions of a burning plasma. Nevertheless, impurity seeding in ELMy $\mathrm{H}$-modes with an ITER-like plasma geometry on JET shows a promising outlook for ITER: (a) a feedback controlled constant radiation level and reduced ELM size at (b) high density, (c) high confinement quality, and (d) $\beta_{N}$ values as needed for ITER, all promising and needed properties for application on ITER. As ITER plasmas will be hotter than the ones obtained on JET up to now, further adjustments will be required with respect to the type of impurity, e.g., the use of krypton could be envisaged, or a mix of impurities such as $\mathrm{N}_{2}$ and $\mathrm{Ar} / \mathrm{Kr}$. Future experiments will have to show the applicability of such proposals.

The most serious restriction for the use of impurity seeding in fusion plasmas is the dilution of fuel ions. To date, the lack of theoretical models to predict anomalous impurity transport is still the largest problem and prevents a detailed quantitative prediction for fusion plasmas with a radiating boundary. Therefore, experimental methods for an active control of impurity transport are of paramount importance. In this context, experiments with radiating plasmas in the TEXTOR tokamak now concentrate on the influence of magnetic perturbation fields at the plasma edge on impurity transport. The recently installed dynamic ergodic divertor (Ref. 53) provides a worldwide unique tool for these studies.

\section{ACKNOWLEDGMENTS}

The TEXTOR team is gratefully acknowledged for providing excellent experimental conditions. We wish to thank $M$. Brix, P. Dumortier, A. Krämer-Flecken, H. R. Koslowski, M. Lehnen, A. Pospieszczyk, J. Rapp, B. Schweer, and G. van Wassenhove for providing valuable data and for clarifying discussions.

\section{REFERENCES}

1. J. JAQUINOT and the JET TEAM, Plasma Phys. Control. Fusion, 41, A13 (1999).

2. Y. SHIMOMURA et al., Nucl. Fusion, 41, 3, 309 (2001). 
3. A. GIBSON and M. L. WATKINS, Proc. 8th European Conf. Controlled Fusion and Plasma Physics, Prague, Czech Republic, Vol. I, p. 31 (1977).

4. K. LACKNER and J. NEUHAUSER, IAEA Technical Mtg. Divertors and Impurity Control, Garching, Germany, p. 58, International Atomic Energy Agency (1981).

5. U. SAMM et al., Proc. 18th Conf. Controlled Fusion and Plasma Physics, Berlin, Germany, Europhysics Conference Abstracts, Vol. 15C, Part III, p. 157, European Physical Society (1991).

6. U. SAMM et al., Plasma Phys. Control. Fusion, 35, B167 (1993).

7. U. SAMM et al., J. Nucl. Mater., 220-222, 25 (1995).

8. U. SAMM et al., J. Nucl. Mater., 196-198, 633 (1992).

9. U. SAMM, M. Z. TOKAR', and B. UNTERBERG, J. Nucl. Mater., 241-243, 827 (1997).

10. E. LAZARUS et al., Nucl. Fusion, 25, 135 (1985).

11. M. BESSENRODT-WEBERPALS, F. X. SÖLDNER, E. R. MÜLLER, H. D. MURMANN, W. POSCHENRIEDER, G. SILLER, and K-H. STEUER, Plasma Phys. Control. Fusion, 34, 443 (1992).

12. J. WINTER et al., Phys. Rev. Lett., 71, 1549 (1993).

13. J. ONGENA et al., Proc. 20th European Conf. Controlled Fusion and Plasma Physics, Lisbon, Portugal, Vol. 17C, Part I, p. 127, European Physical Society (1993).

14. A. M. MESSIAEN et al., Nucl. Fusion, 34, 825 (1994).

15. A. M. MESSIAEN et al., Phys. Rev. Lett., 77, 2487 (1996).

16. A. M. MESSIAEN et al., Phys. Plasmas, 4, 1690 (1997).

17. B. UNTERBERG et al., Plasma Phys. Control. Fusion, 39, B189 (1997).

18. R. R. WEYNANTS et al., Nucl. Fusion, 39, 1637 (1999).

19. M. GREENWALD et al., Nucl. Fusion, 28, 2199 (1988).

20. G. MANK et al., Phys. Rev. Lett., 85, 2312 (2000).

21. B. UNTERBERG et al., Proc. 18th Fusion Energy Conf., Sorrento, Italy, Paper IAEA-CN-77/EX5/2, International Atomic Energy Agency (2000).

22. J. ONGENA et al., Phys. Plasmas, 8, 2188 (2001).

23. A. KALLENBACH et al., Plasma Phys. Control. Fusion, 38, 2097 (1996).

24. G. M. STAEBLER et al., Phys. Rev. Lett., 82, 1692 (1999).

25. M. MURAKAMI et al., Nucl. Fusion, 41, 317 (2001).

26. G. L. JACKSON et al., Nucl. Fusion, 42, 28 (2002).

27. J. ONGENA et al., Proc. 24th European Conf. Controlled Fusion and Plasma Physics, Berchtesgaden, Part IV, p. 1693, European Physical Society (1997)

28. J. ONGENA et al., Plasma Phys. Control. Fusion, 41, A379 (1999).
29. H. KUBO et al., Nucl. Fusion, 41, 227 (2001).

30. J. ONGENA et al., Plasma Phys. Control. Fusion, 43, A11 (2001). 31. P. DUMORTIER et al., Plasma Phys. Control. Fusion, 44, 1845 (2002).

32. J. ONGENA et al., Nucl. Fusion, 44, 124 (2004).

33. B. UNTERBERG et al., J. Nucl. Mater., 266-299, 75 (1999).

34. M. Z. TOKAR', Contrib. Plasma Phys., 34, 2/3, 344 (1994).

35. R. JASPERS et al., Proc. 26th Conf. Plasma Physics and Controlled Fusion, Maastricht, The Netherlands, Europhysics Conference Abstracts, Vol. 23J, p. 601, European Physical Society (1999).

36. G. VAN WASSENHOVE et al., Proc. 26th Conf. Plasma Physics and Controlled Fusion, Maastricht, The Netherlands, Europhysics Conference Abstracts, Vol. 23J, p. 741, European Physical Society (1999).

37. M. Z. TOKAR', Plasma Phys. Control. Fusion, 36, 1819 (1994).

38. G. F. MATTHEWS et al., J. Nucl. Mater., 241-243, 450 (1997). 39. H. R. KOSLOWSKI et al., Nucl. Fusion, 40, 821 (2000).

40. M. Z. TOKAR' et al., Plasma Phys. Control. Fusion, 41, L9 (1999). 41. R. D. SYDORA, V. K. DECYK, and J. M. DAWSON, Plasma Phys. Control. Fusion, 38, 281 (1996).

42. R. D. SYDORA et al., Proc. 18th Fusion Energy Conf., Sorrento, Italy, Paper IAEA-CN-77/THP1/27, International Atomic Energy Agency (2000).

43. J. WEILAND, Collective Modes in Inhomogeneous Plasma, Plasma Physics Series, IOP Publishing Ltd., Bristol, United Kingdom (2000).

44. M. Z. TOKAR', J. ONGENA, B. UNTERBERG, and R. R. WEYNANTS, Phys. Rev. Lett., 84, 5, 895 (2000).

45. J. NILSSON and J. WEILAND, Nucl. Fusion, 34, 803 (1994).

46. M. Z. TOKAR', R. JASPERS, and B. UNTERBERG, Nucl. Fusion, 38, L961 (1998).

47. G. R. McKEE et al., Phys. Rev. Lett., 84, 1922 (2000).

48. J. RAPP et al., Nucl. Fusion, 44, 312 (2004).

49. G. L. JACKSON et al., Proc. 28th Conf. Plasma Physics and Controlled Fusion, Funchal, Portugal, Europhysics Conference Abstracts, Vol. 25A, p. 993, European Physical Society (2001).

50. M. Z. TOKAR' et al., Plasma Phys. Control. Fusion, 44, 1903 (2002).

51. B. UNTERBERG et al., Plasma Phys. Control. Fusion, 46, A241 (2004).

52. B. UNTERBERG, D. KALUPIN, and M. Z. TOKAR' (to be published).

53. K. H. FINKEN, Ed., Dynamic Ergodic Divertor (special issue) Fusion Eng. Des., 37, 335 (1997). 